\title{
Bäcklund transformation and smooth multisoliton solutions for a modified Camassa-Holm equation with cubic nonlinearity
}

\author{
Yoshimasa Matsunda \\ Division of Applied Mathematical Science, \\ Graduate School of Science and Engineering \\ Yamaguchi University, Ube, Yamaguchi 755-8611, Japan
}

\begin{abstract}
We present a compact parametric representation of the smooth bright multisolution solutions for the modified Camassa-Holm $(\mathrm{mCH})$ equation with cubic nonlinearity. We first transform the $\mathrm{mCH}$ equation to an associated $\mathrm{mCH}$ equation through a reciprocal transformation and then find a novel Bäcklund transformation between solutions of the associated $\mathrm{mCH}$ equation and a model equation for shallow-water waves (SWW) introduced by Ablowitz at al. We combine this result with the expressions of the multisoliton solutions for the SWW and modified Korteweg-de Vries equations to obtain the multisoliton solutions of the $\mathrm{mCH}$ equation. Subsequently, we investigate the properties of the one- and two-soliton solutions as well as the general multisoliton solutions. We show that the smoothness of the solutions is assured only if the amplitude parameters of solitons satisfy certain conditions. We also find that at a critical value of the parameter beyond which the solution becomes singular, the soliton solution exhibits a different feature from that of the peakon solution of the $\mathrm{CH}$ equation. Then, by performing an asymptotic analysis for large time, we obtain the formula for the phase shift and confirm the solitonic nature of the multisoliton solutions. Last, we use the Bäcklund transformation to derive an infinite number of conservation laws of the $\mathrm{mCH}$ equation.
\end{abstract}

\footnotetext{
${ }^{\mathrm{a}}$ E-mail address: matsuno@yamaguchi-u.ac.jp
} 


\section{INTRODUCTION}

A modified Camassa-Holm $(\mathrm{mCH})$ equation with cubic nonlinearity is a current interest in the theory of solitons. It may be written in the form

$$
m_{t}+\left[m\left(u^{2}-u_{x}^{2}\right)\right]_{x}=0, \quad m=u-u_{x x},
$$

where $u=u(x, t)$ is a real-valued function of time $t$ and a spatial variable $x$, and the subscripts $x$ and $t$ appended to $m$ and $u$ denote partial differentiation. The $\mathrm{mCH}$ equation was found independently by several researchers using a procedure that generates new integrable systems from known integrable bi-hamiltonian systems. ${ }^{1-3}$. It is well-known that the method yields the $\mathrm{CH}$ equation ${ }^{4}$ when applied to the Korteweg-de Vries (KdV) equation whereas it yields Eq. (1.1) when applied to the modified KdV (mKdV) equation. ${ }^{1-3}$ It also arises from the physical system like nonlinear water waves. ${ }^{1,5}$ Although a large number of works have been devoted to the $\mathrm{CH}$ equation, ${ }^{6}$ the study of the $\mathrm{mCH}$ equation is still under way. Specifically, a recent work by Qiao reveals that it admits W/M-shape peaked solitons unlike the peakon solutions of the $\mathrm{CH}$ equation. ${ }^{5,7}$ Quite recently, smooth dark soliton solutions of the integrable hierarchy including the $\mathrm{mCH}$ equation were obtained for the variable $m$ using the inverse scattering transform (IST) method and the properties of the one- and two-soliton solutions were explored. ${ }^{8}$

The purpose of the present paper is to construct the bright $N$-soliton solutions of the $\mathrm{mCH}$ equation under the boundary condition $u \rightarrow u_{0}$ as $|x| \rightarrow \infty$, where $N$ is an arbitrary positive integer and $u_{0}$ is a positive constant. We employ an exact method of solution which worked effectively for constructing the $N$-soliton solutions of the $\mathrm{CH}$ and Degaperis-Procesi equations. ${ }^{9-11}$ As a byproduct, an infinite number of conservation laws are produced in a simple manner without recourse to the IST.

The paper is organized as follows. In Sec. II, the $\mathrm{mCH}$ is transformed to an associated $\mathrm{mCH}$ equation by introducing a reciprocal transformation. We find a remarkable Bäcklund transformation connecting solutions of the associated $\mathrm{CH}$ equation with those of a model equation for shallow-water waves (SWW equation for short) introduced by Ablowitz et al. ${ }^{12}$ This allows us to obtain the parametric representation for the bright $N$-soliton solution of the $\mathrm{mCH}$ equation when coupled with the $N$-soliton solutions of 
the SWW and mKdV equations. In Sec. III, the properties of the one- and two soliton solutions are investigated in detail. In particular, we obtain a condition necessary for the existence of smooth solitons and show that there exists a critical value of the amplitude parameter of the soliton beyond which the solution becomes singular. Subsequently, the asymptotic behavior of the $N$-soliton solution is briefly discussed, whereby we derive the formula for the phase shift and confirm the solitonic behavior of the solution. In Sec. IV, an infinite number of conservation laws are derived simply through the Bäcklund transformation and their explicit expressions are presented for the first few members. Section $\mathrm{V}$ is devoted to concluding remarks.

\section{CONSTRUCTION OF MULTISOLITON SOLUTIONS}

\section{A. Associated $\mathrm{mCH}$ equation}

We first introduce a coordinate transformation $(x, t) \rightarrow(y, \tau)$ by

$$
d y=m d x-m\left(u^{2}-u_{x}^{2}\right) d t, \quad d \tau=d t
$$

subjected to the restriction $m>0$. Accordingly, the $x$ and $t$ derivatives can be rewritten as

$$
\frac{\partial}{\partial x}=m \frac{\partial}{\partial y}, \quad \frac{\partial}{\partial t}=\frac{\partial}{\partial \tau}-m\left(u^{2}-u_{x}^{2}\right) \frac{\partial}{\partial y} .
$$

It then follows from $(2.1 \mathrm{~b})$ that the variable $x=x(y, \tau)$ satisfies a system of linear partial differential equations (PDEs)

$$
x_{y}=\frac{1}{m}, \quad x_{\tau}=u^{2}-m^{2} u_{y}^{2} .
$$

We apply the transformation $(2.1 \mathrm{~b})$ to the $\mathrm{mCH}$ equation and find that it can be recast into the form

$$
m_{\tau}+2 m^{3} u_{y}=0
$$

The integrability condition $x_{\tau y}=x_{y \tau}$ of the system (2.2) is satisfied automatically by virtue of Eq. (2.3). Using (2.3) and the relation $u_{x x}=m^{2} u_{y y}+m m_{y} u_{y}$, the variable $u\left(=m+u_{x x}\right)$ is expressed in terms of $m$ as

$$
u=m+\frac{1}{2} m\left(\frac{1}{m}\right)_{\tau y} .
$$


Last, if we define the new variable $p$ by $p=1 / m$ and substitute $u$ from (2.4) into (2.3), we obtain a nonlinear PDE for $p$ :

$$
p p_{\tau y y}-p_{y} p_{\tau y}-p^{3} p_{\tau}-2 p_{y}=0
$$

This evolution equation for $p$ in the independent variables $\tau$ and $y$ is the reciprocal transformation for the $\mathrm{mCH}$ equation. We call it the associated $\mathrm{mCH}$ equation.

\section{B. Multisoliton solutions}

The main result in this paper is given by the following theorem:

Theorem 2.1: The $m C H$ equation (1.1) admits the parametric representation for the $N$-soliton solution

$$
\begin{gathered}
u=u(y, \tau)=u_{0}-\left(\ln f^{\prime} f\right)_{\tau y}, \\
x=x(y, \tau)=\frac{y}{u_{0}}+u_{0}^{2} \tau+2 \ln \frac{f^{\prime}}{f}+d,
\end{gathered}
$$

where $f$ and $f^{\prime}$ are tau-functions given by

$$
\begin{gathered}
f=\sum_{\mu=0,1} \exp \left[\sum_{i=1}^{N} \mu_{i} \xi_{i}+\sum_{1 \leq i<j \leq N} \mu_{i} \mu_{j} \gamma_{i j}\right], \\
f^{\prime}=\sum_{\mu=0,1} \exp \left[\sum_{i=1}^{N} \mu_{i}\left(\xi_{i}-\phi_{i}\right)+\sum_{1 \leq i<j \leq N} \mu_{i} \mu_{j} \gamma_{i j}\right],
\end{gathered}
$$

with

$$
\begin{gathered}
\xi_{i}=k_{i}\left[y-\frac{2 u_{0}^{3}}{1-\left(u_{0} k_{i}\right)^{2}} \tau-\left(\frac{6}{u_{0}^{2}}-4 k_{i}^{2}\right) s-y_{i 0}\right], \quad(i=1,2, \ldots, N), \\
\mathrm{e}^{-\phi_{i}}=\frac{1-u_{0} k_{i}}{1+u_{0} k_{i}}, \quad(i=1,2, \ldots, N), \\
\mathrm{e}^{\gamma_{i j}}=\left(\frac{k_{i}-k_{j}}{k_{i}+k_{j}}\right)^{2}, \quad(i, j=1,2, \ldots, N ; i \neq j) .
\end{gathered}
$$

Here, $k_{i}$ and $y_{i 0}$ are the amplitude and phase parameters of the ith soliton, respectively, $d$ is an arbitrary constant, $s$ is an auxiliary time variable and the notation $\sum_{\mu=0,1}$ implies the summation over all possible combination of $\mu_{1}=0,1, \mu_{2}=0,1, \ldots, \mu_{N}=0,1$. 
The parametric solution provided by Theorem 2.1 would give rise to the smooth bright $N$-soliton solution on a constant background $u=u_{0}$ if the conditions $0<u_{0} k_{i}<\sqrt{3} / 2(i=$ $1,2, \ldots, N)$ are imposed on the amplitude parameters. The variable $s$ introduced in (2.10a) plays the role of the time variable for both the $\mathrm{KdV}$ and $\mathrm{mKdV}$ equations. It can be set to zero after all the computations have been completed.

Now, Theorem 2.1 follows straightforwardly from Propositions 2.1 and 2.2 below when combined with the system (2.2), which we shall now demonstrate.

Proposition 2.1: The variables $p, q$ and $r$ defined by

$$
\begin{gathered}
p=\frac{1}{u_{0}}+2\left(\ln \frac{f^{\prime}}{f}\right)_{y}, \\
q=-2(\ln f)_{y y} \\
r=\frac{1}{u_{0}^{2}}-8(\ln f)_{y y}
\end{gathered}
$$

satisfy the nonlinear PDEs

$$
\begin{aligned}
p_{s}+6 p^{2} p_{y}-4 p_{\text {yyy }} & =0, \\
q_{\tau}+2 u_{0}^{3} q_{y}+4 u_{0}^{2} q q_{\tau}+2 u_{0}^{2} q_{y} \partial_{y}^{-1} q_{\tau}-u_{0}^{2} q_{\tau y y} & =0, \quad\left(\partial_{y}^{-1}=-\int_{y}^{\infty} d y\right), \\
r_{s}+6 r r_{y}-4 r_{y y y} & =0,
\end{aligned}
$$

respectively. Then, the variables $r$ and $q$ are expressed in terms of $p$ as

$$
\begin{gathered}
r=p^{2}+2 p_{y}, \\
q=\frac{p_{y}}{2}+\frac{p^{2}}{4}-\frac{1}{4 u_{0}^{2}} .
\end{gathered}
$$

Proof: Equation (2.14) is the mKdV equation of defocusing type. It exhibits the dark $N$-soliton solution (2.11) with the tau-functions (2.8) and (2.9). ${ }^{13,14}$ Equation (2.15) is a SWW equation for which the $N$-soliton solution is given by (2.12) with the tau-function (2.8) whereas Eq. (2.16) is the KdV equation whose tau-function has the same functional form as that of the SWW equation except the time dependence. ${ }^{12,15}$ The latter follows 
from the fact that the SWW equation belongs to a member of the integrable hierarchy of the KdV equation. ${ }^{12}$

The relation (2.17) is the Miura transformation ${ }^{16}$ which connects solutions of the mKdV equation with those of the KdV equation. Actually, a direct substitution of (2.17) into the left-hand side of (2.16) reveals that

$$
r_{s}+6 r r_{y}-4 r_{y y y}=2\left(\frac{\partial}{\partial y}+p\right)\left(p_{s}+6 p^{2} p_{y}-4 p_{y y y}\right) .
$$

The relation (2.18) stems simply from (2.12), (2.13) and (2.17) by eliminating the tau-function $f$.

The relation (2.18) is a Bäcklund transformation between solutions of the associated $\mathrm{mCH}$ equation and the SWW equation, as will be shown by Proposition 2.2. If one substitutes (2.11)-(2.13) into (2.17) and (2.18), then one can see that they reduce to the following bilinear equation for $f$ and $f^{\prime}$ :

$$
f_{y y}^{\prime} f-2 f_{y}^{\prime} f_{y}+f^{\prime} f_{y y}+\frac{1}{u_{0}}\left(f_{y}^{\prime} f-f^{\prime} f_{y}\right)=0 .
$$

The above equation is a constituent of the system of bilinear equations for the $\mathrm{mKdV}$ equation and hence the tau-functions $f$ and $f^{\prime}$ from (2.8) and (2.9) solve Eq. (2.20). ${ }^{17}$

Proposition 2.2: Let

$$
\begin{gathered}
Q=q_{\tau}+2 u_{0}^{3} q_{y}+4 u_{0}^{2} q q_{\tau}+2 u_{0}^{2} q_{y} \partial_{y}^{-1} q_{\tau}-u_{0}^{2} q_{\tau y y}, \\
R=\frac{p_{\tau y y}}{p}-\frac{p_{y} p_{\tau y}}{p^{2}}-p p_{\tau}-2 \frac{p_{y}}{p^{2}} .
\end{gathered}
$$

Then, $Q$ and $R$ are connected by the relation

$$
Q=-\frac{u_{0}^{2}}{2}\left(\frac{\partial}{\partial y}+p\right) \frac{\partial}{\partial y}\left(p \partial_{y}^{-1} R\right)
$$

under the transformation (2.18).

Proof: Substituting (2.18) into (2.21), one can recast $Q$ into the form

$$
Q=\frac{u_{0}^{2}}{2}\left[\left(p_{y y}+p p_{y}\right)\left\{2 u_{0}+\partial_{y}^{-1}\left(p p_{\tau}\right)\right\}+p^{2} p_{\tau y}+3 p p_{\tau} p_{y}+p^{3} p_{\tau}-p_{\tau y y y}-p p_{\tau y y}\right] .
$$


On the other hand, integration of (2.22) with respect to $y$ under the boundary condition $p \rightarrow 1 / u_{0},|y| \rightarrow \infty$ gives

$$
2 u_{0}+\partial_{y}^{-1}\left(p p_{\tau}\right)=-\partial_{y}^{-1} R+\frac{1}{p}\left(p_{\tau y}+2\right),
$$

whereas a direct computation using (2.22) leads to

$$
\frac{\left(p^{2} R\right)_{y}}{p}+p^{2} R=-p^{2} p_{\tau y}-3 p p_{\tau} p_{y}-p^{3} p_{\tau}+p_{\tau y y y}+p p_{\tau y y}-\frac{1}{p}\left(p_{y y}+p p_{y}\right)\left(p_{\tau y}+2\right) .
$$

The relation (2.22) follows by introducing above two expressions into $Q$. Actually,

$$
Q=-\frac{u_{0}^{2}}{2}\left[\left(p_{y y}+p p_{y}\right) \partial_{y}^{-1} R+p R_{y}+\left(2 p_{y}+p^{2}\right) R\right]=-\frac{u_{0}^{2}}{2}\left(\frac{\partial}{\partial y}+p\right) \frac{\partial}{\partial y}\left(p \partial_{y}^{-1} R\right) .
$$

This completes the proof.

It is obvious from (2.23) that the equation $R=0$ yields the equation $Q=0$. But, the converse statement is not true in general. For the $N$-soliton solution (2.11) which satisfies the boundary condition $p \rightarrow p_{0}\left(=1 / u_{0}\right),|y| \rightarrow \infty$, however, $p$ is a solution of Eq. (2.5), or $R=0$ if $q$ from (2.12) satisfies Eq. (2.15), or $Q=0$. To see this, assume first that $Q=0$. Integrating the resultant equation from (2.23) with respect to $y$ and using the boundary condition $p \rightarrow p_{0}, y \rightarrow+\infty$, one obtains

$$
\frac{\partial}{\partial y}\left(p \partial_{y}^{-1} R\right)=R_{0}(\tau) \exp \left[\int_{y}^{\infty}\left(p-p_{0}\right) d y-p_{0} y\right]
$$

where $R_{0}(\tau)$ is an integration constant. A slight modification of the relation for $\partial_{y}^{-1} R$ derived in the proof of Proposition 2.2 gives

$$
\partial_{y}^{-1} R=\frac{p_{\tau y}}{p}+\frac{1}{2} \frac{\partial}{\partial \tau} \int_{y}^{\infty}\left(p^{2}-p_{0}^{2}\right) d y+\frac{2}{p}-\frac{2}{p_{0}} .
$$

As confirmed easily by integrating (2.18) with respect to $y$ and using (2.12), the integral $\int_{-\infty}^{\infty}\left(p^{2}-p_{0}^{2}\right) d y$ becomes a constant independent of $\tau$. It turns out that $\lim _{y \rightarrow-\infty} \partial_{y}^{-1} R=0$. With this fact in mind, the left-hand side of (2.24) vanishes in the limit of $y \rightarrow-\infty$. On the other hand, the right-hand side diverges in this limit. Thus, one must put $R_{0}=0$, which yields $\left(p \partial_{y}^{-1} R\right)_{y}=0$. Integrating again with respect to $y$, one has $\partial_{y}^{-1} R=R_{1}(\tau) / p$, where $R_{1}(\tau)$ is an integration constant. In view of the boundary condition, this constant 
must be zero, and hence $\partial_{y}^{-1} R=0$. Differentiating this relation by $y$, one finally arrives at the relation $R=0$.

We are now ready for proving Theorem 1.1.

Proof: First, referring to (2.2) and (2.11), one obtains

$$
x_{y}=p=\frac{1}{u_{0}}+2\left(\ln \frac{f^{\prime}}{f}\right)_{y} .
$$

Integrating this relation with respect to $y$ leads to

$$
x=\frac{y}{u_{0}}+\ln \frac{f^{\prime}}{f}+d(\tau),
$$

where $d(\tau)$ is an integration constant. Differentiate the above expression by $\tau$ and use (2.2) to find the relation

$$
x_{\tau}=2\left(\ln \frac{f^{\prime}}{f}\right)_{\tau}+d^{\prime}(\tau)=u^{2}-m^{2} u_{y}^{2} .
$$

This expression reduces, upon taking the limit $y \rightarrow \infty$ and substituting the limiting values $u \rightarrow u_{0}, u_{y} \rightarrow 0,\left[\ln \left(f^{\prime} / f\right)\right]_{\tau} \rightarrow 0$, to $d^{\prime}(\tau)=u_{0}^{2}$, which gives $d(\tau)=u_{0}^{2} \tau+d$ after integrating with respect to $\tau$. This proves (2.7).

It now follows by integrating Eq. (2.3) with respect to $y$ and substituting $p^{2}$ from (2.18) into the resultant expression that

$$
u=u_{0}+\frac{1}{4} \int_{-\infty}^{y}\left(p^{2}\right)_{\tau} d y=u_{0}+\int_{-\infty}^{y} q_{\tau} d y-\frac{1}{2} p_{\tau} .
$$

Last, substitution of (2.11) and (2.12) into the above expression yields

$$
u=u_{0}-2(\ln f)_{\tau y}-\left(\ln \frac{f^{\prime}}{f}\right)_{\tau y}=u_{0}-\left(\ln f^{\prime} f\right)_{\tau y},
$$

which is just (2.6).

\section{PROPERTIES OF SOLUTIONS}

\section{A. One-soliton solution}

The tau-functions $f$ and $f^{\prime}$ corresponding to the one-soliton solution are given by (2.8), (2.9) and (2.10) with $N=1$. They read

$$
f=1+\mathrm{e}^{\xi},
$$




$$
f^{\prime}=1+\mathrm{e}^{\xi-\phi}
$$

with

$$
\begin{gathered}
\xi=k\left(y-\tilde{c} \tau-y_{0}\right), \quad \tilde{c}=\frac{2 u_{0}^{3}}{1-\left(u_{0} k\right)^{2}}, \\
\mathrm{e}^{-\phi}=\frac{1-u_{0} k}{1+u_{0} k},
\end{gathered}
$$

where we have put $\xi=\xi_{1}, \phi=\phi_{1}, k=k_{1}$ and $y_{0}=y_{10}$ for simplicity and set $s=0$. We assume $k>0$ hereafter.

The parametric representation of the one-soliton solution follows by introducing (3.1) and (3.2) into (2.6) and (2.7). It may be written in the form

$$
\begin{gathered}
u=u_{0}+\frac{k^{2} \tilde{c}\left\{a \cosh \left(\xi-\xi_{0}\right)+1\right\}}{\left\{\cosh \left(\xi-\xi_{0}\right)+a\right\}^{2}}, \\
x-c t-x_{0}=\frac{\xi}{u_{0} k}+2 \ln \left(\frac{\sqrt{1-\alpha} \mathrm{e}^{\xi-\xi_{0}}+\sqrt{1+\alpha}}{\sqrt{1+\alpha} \mathrm{e}^{\xi-\xi_{0}}+\sqrt{1-\alpha}}\right),
\end{gathered}
$$

where

$$
\begin{aligned}
\alpha=u_{0} k, \quad a & =\frac{1}{\sqrt{1-\left(u_{0} k\right)^{2}}}, \quad \xi_{0}=\frac{1}{2} \ln \left(\frac{1+u_{0} k}{1-u_{0} k}\right), \\
c & =\frac{\tilde{c}}{u_{0}}+u_{0}^{2}=\frac{u_{0}^{2}\left\{3-\left(u_{0} k\right)^{2}\right\}}{1-\left(u_{0} k\right)^{2}},
\end{aligned}
$$

and we have put $d=x_{0}-y_{0} / u_{0}-\ln [(1-\alpha) /(1+\alpha)]$. Note that $c$ from $(3.4 \mathrm{~d})$ is the velocity of the soliton in the $(x, t)$ coordinate system. The smoothness of the soliton solution is assured by the condition $m=1 / p>0$ (see (2.1a) and (2.1b)) which imposes certain restriction on the parameter $\alpha$. To show this explicitly, we compute $x_{y}(=p)$ from (3.4b) and obtain

$$
p=\frac{1}{u_{0}}-\frac{2 k \alpha}{\sqrt{1-\alpha^{2}} \cosh \left(\xi-\xi_{0}\right)+1} .
$$

Recall that $p$ is a dark soliton solution of the $\mathrm{mKdV}$ equation (2.14). The required condition is then found to be satisfied if $1 / u_{0}-2 k \alpha /\left(\sqrt{1-\alpha^{2}}+1\right)>0$. Thus, the parameter $\alpha$ must lie in the interval

$$
0<\alpha<\frac{\sqrt{3}}{2} \text {. }
$$




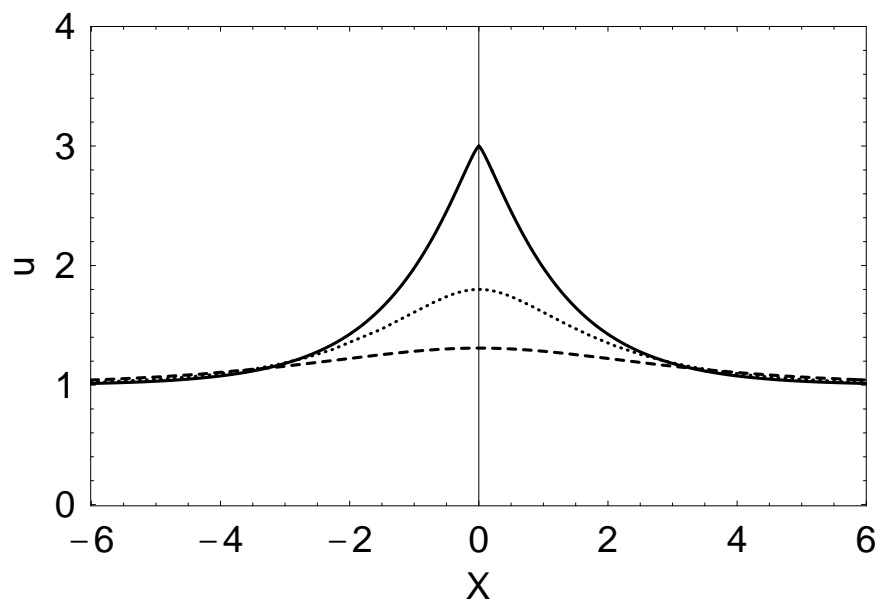

FIG. 1. The profile of smooth solitons. $\alpha=0.5$ (dashed curve), $\alpha=0.7$ (dotted curve), $\alpha=\sqrt{3} / 2$ (solid curve).

One can see from (3.4a) and (3.4b) that the one-soliton solution represents a bright soliton on a constant background $u=u_{0}$ whose center position $x_{c}$ is located at $x_{c}=$ $c t+x_{0}+\xi_{0} /\left(u_{0} k\right)\left(\xi=\xi_{0}\right)$. In view of this observation, the amplitude of the soliton with respect to the background field, which we denote by $A$, amounts to

$$
A=\frac{2 u_{0}}{\sqrt{1-\alpha^{2}}}\left(1-\sqrt{1-\alpha^{2}}\right) .
$$

Eliminating the parameter $\alpha$ from (3.4d) and (3.7) leads to the amplitude-velocity relation of the soliton

$$
A=\sqrt{2\left(c-u_{0}^{2}\right)}-2 u_{0}
$$

The inequality (3.6) restricts possible values of $c$ and $A$. Explicitly,

$$
3 u_{0}^{2}<c<9 u_{0}^{2}, \quad 0<A<2 u_{0}
$$

If the condition (3.6) is satisfied, then the one-soliton solution represents a smooth bright solitary wave travelling to the right with velocity $c$ and amplitude $A$. As $\alpha$ increases, the amplitude grows and the width narrows. This observation is illustrated in Fig. 1, whereby the profile of smooth solitons with a background $u_{0}=1$ is depicted agianst the stationary variable $X=x-c t-x_{0}$ for three representative values of $\alpha$. Of particular interest is the limiting profile of the soliton when the parameter $\alpha$ tends to the upper 


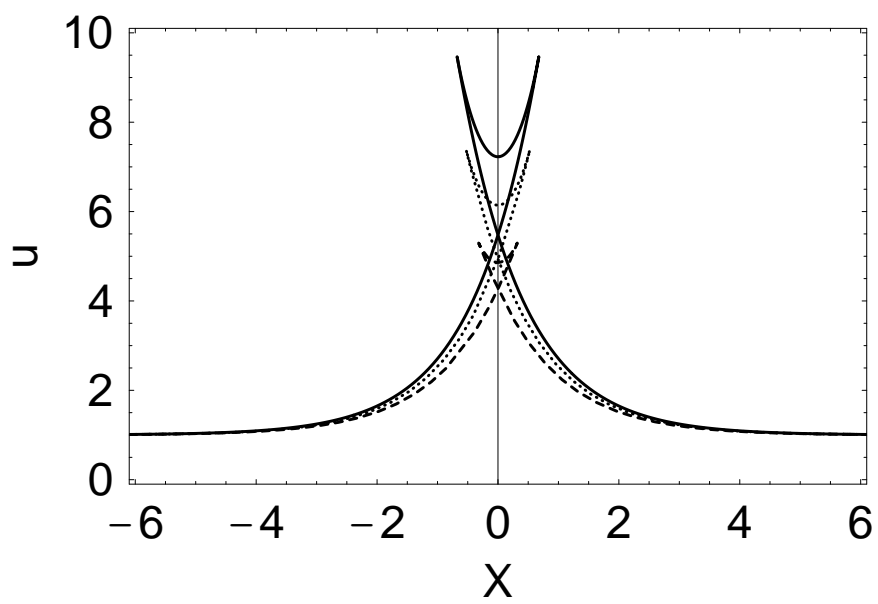

FIG. 2. The profile of singular solitons. $\alpha=0.94$ (dashed curve), $\alpha=0.96$ (dotted curve), $\alpha=0.97$ (solid curve).

limit $\alpha=\sqrt{3} / 2(\simeq 0.866)$ of the inequality (3.6) (see solid curve in Fig. 1$)$. In this limit, the smoothness of the profile would be lost at the center position (or the crest) of the soliton. The typical profile is also depicted in Fig. 2 for singular solitons which violate the condition (3.6).

To explore the feature of the solution in more detail, we shall investigate the profile of the soliton near its crest. To this end, we first derive the approximate expression of $X$ from (3.4b). If we choose the phase parameter of the soliton appropriately such that $\xi=\xi_{0}$ corresponds to $X=0$, then the power series expansion of $X$ with respect to $\xi-\xi_{0}$ is found to be as

$$
X=-\frac{1}{\alpha}\left(1-2 \sqrt{1-\alpha^{2}}\right)\left(\xi-\xi_{0}\right)+\frac{1}{3} \frac{\sqrt{1-\alpha^{2}}}{\alpha^{3}}\left(1-\sqrt{1-\alpha^{2}}\right)^{2}\left(\xi-\xi_{0}\right)^{3}+O\left(\left(\xi-\xi_{0}\right)^{5}\right) \text {. }
$$

In particular, for $\alpha=\sqrt{3} / 2,(3.10)$ reduces to

$$
X=\frac{\sqrt{3}}{27}\left(\xi-\xi_{0}\right)^{3}+O\left(\left(\xi-\xi_{0}\right)^{5}\right) .
$$

On the other hand, the expansion of $u$ from $(3.4 \mathrm{a})$ reads

$$
u=u_{0}\left[1+\frac{6}{1+a}+\frac{3(-2+a)}{(1+a)^{2}}\left(\xi-\xi_{0}\right)^{2}+\frac{16-13 a+a^{2}}{4(1+a)^{3}}\left(\xi-\xi_{0}\right)^{4}+O\left(\left(\xi-\xi_{0}\right)^{6}\right)\right]
$$


which simplifies to

$$
u=u_{0}\left[3-\frac{1}{18}\left(\xi-\xi_{0}\right)^{4}+O\left(\left(\xi-\xi_{0}\right)^{6}\right)\right] .
$$

when $a=2(\alpha=\sqrt{3} / 2)$. Note that the coefficient of $\left(\xi-\xi_{0}\right)^{2}$ in the expansion (3.12) vanishes for this specific value of $a$. By inserting $\xi-\xi_{0}$ from (3.11) into (3.13), we find that the profile of the soliton near the crest is approximated by the expression

$$
u \sim u_{0}\left[3-\frac{3^{4 / 3}}{2} X^{4 / 3}\right] .
$$

Thus, we can see that the smoothness of the solution is lost at the crest $X=0$. More precisely, $u$ has only a continuous first derivative near the crest and the $n$th derivative does not exists for $n \geq 2$ at the crest. This novel feature is in striking contrast to the appearance of the singularity (or discontinuous first derivative at the crest) in the peakon solution which can be deduced from the smooth soliton solution of the $\mathrm{CH}$ equation through an appropriate limiting process. ${ }^{18,19}$

\section{B. Two-soliton solution}

The tau-functions $f$ and $f^{\prime}$ for the two-soliton solution can be written as

$$
\begin{gathered}
f=1+\mathrm{e}^{\xi_{1}}+\mathrm{e}^{\xi_{2}}+\left(\frac{k_{1}-k_{2}}{k_{1}+k_{2}}\right)^{2} \mathrm{e}^{\xi_{1}+\xi_{2}}, \\
f^{\prime}=1+\mathrm{e}^{\xi_{1}-\phi_{1}}+\mathrm{e}^{\xi_{2}-\phi_{2}}+\left(\frac{k_{1}-k_{2}}{k_{1}+k_{2}}\right)^{2} \mathrm{e}^{\xi_{1}+\xi_{2}-\phi_{1}-\phi_{2}},
\end{gathered}
$$

where

$$
\begin{gathered}
\xi_{i}=k_{i}\left(y-\tilde{c}_{i} \tau-y_{i 0}\right), \quad \tilde{c}_{i}=\frac{2 u_{0}^{3}}{1-\left(u_{0} k_{i}\right)^{2}}, \quad(i=1,2), \\
\mathrm{e}^{-\phi_{i}}=\frac{1-u_{0} k_{i}}{1+u_{0} k_{i}}, \quad(i=1,2) .
\end{gathered}
$$

We investigate the asymptotic behavior of the two-soliton solution for large time and show that it describes the elastic interaction of two bright solitons on a background field $u=u_{0}$. To this end, let $c_{i}(i=1,2)$ be the velocity of the $i$ the soliton in the $(x, t)$ coordinate system and assume that $0<c_{2}<c_{1}$. In addition, we impose the conditions $0<u_{0} k_{i}<\sqrt{3} / 2,(i=1,2)$ to assure the smoothness of the solution. 
First, we take the limit $t \rightarrow-\infty$ with $\xi_{1}$ being fixed. In this limit, $\xi_{2} \rightarrow-\infty$. Then, the leading-order asymptotics of the tau-functions are given by

$$
\begin{gathered}
f \sim 1+\mathrm{e}^{\xi_{1}}, \\
f^{\prime}=1+\mathrm{e}^{\xi_{1}-\phi_{1}} .
\end{gathered}
$$

It folows from (2.6) and (2.7), (3.16a), and (3.16b) that

$$
\begin{gathered}
u \sim u_{0}+\frac{k_{1}^{2} \tilde{c}_{1}\left\{a_{1} \cosh \left(\xi_{1}-\xi_{10}\right)+1\right\}}{\left\{\cosh \left(\xi_{1}-\xi_{10}\right)+a_{1}\right\}^{2}}, \\
x-c_{1} t-x_{10} \sim \frac{\xi_{1}}{u_{0} k_{1}}+2 \ln \left(\frac{\sqrt{1-\alpha_{1}} \mathrm{e}^{\xi_{1}-\xi_{10}}+\sqrt{1+\alpha_{1}}}{\sqrt{1+\alpha_{1}} \mathrm{e}^{\xi_{1}-\xi_{10}}+\sqrt{1-\alpha_{1}}}\right),
\end{gathered}
$$

where

$$
\begin{aligned}
\alpha_{1}=u_{0} k_{1}, \quad a_{1} & =\frac{1}{\sqrt{1-\left(u_{0} k_{1}\right)^{2}}}, \quad \xi_{10}=\frac{1}{2} \ln \left(\frac{1+u_{0} k_{1}}{1-u_{0} k_{1}}\right), \\
c_{1} & =\frac{\tilde{c}_{1}}{u_{0}}+u_{0}^{2}=\frac{u_{0}^{2}\left\{3-\left(u_{0} k_{1}\right)^{2}\right\}}{1-\left(u_{0} k_{1}\right)^{2}},
\end{aligned}
$$

In the limit $t \rightarrow+\infty$, on the other hand, $\xi_{2} \rightarrow+\infty$. The expressions corresponding to $(3.16 \mathrm{a})-(3.17 \mathrm{~b}) \mathrm{read}$

$$
\begin{gathered}
f \sim \mathrm{e}^{\xi_{2}}\left[1+\left(\frac{k_{1}-k_{2}}{k_{1}+k_{2}}\right)^{2} \mathrm{e}^{\xi_{1}}\right], \\
f^{\prime}=\mathrm{e}^{\xi_{2}-\phi_{2}}\left[1+\left(\frac{k_{1}-k_{2}}{k_{1}+k_{2}}\right)^{2} \mathrm{e}^{\xi_{1}-\phi_{1}}\right] . \\
u \sim u_{0}+\frac{k_{1}^{2} \tilde{c}_{1}\left\{a_{1} \cosh \left(\xi_{1}-\xi_{10}+\delta_{1}^{(+)}\right)+1\right\}}{\left\{\cosh \left(\xi_{1}-\xi_{10}+\delta_{1}^{(+)}\right)+a_{1}\right\}^{2}}, \\
x-c_{1} t-x_{10} \sim \frac{\xi_{1}}{u_{0} k_{1}}+2 \ln \left(\frac{\sqrt{1-\alpha_{1}} \mathrm{e}^{\xi_{1}-\xi_{10}+\delta_{1}^{(+)}}+\sqrt{1+\alpha_{1}}}{\sqrt{1+\alpha_{1}} \mathrm{e}^{\xi_{1}-\xi_{10}+\delta_{1}^{(+)}}+\sqrt{1-\alpha_{1}}}\right)-2 \phi_{2},
\end{gathered}
$$

where

$$
\delta_{1}^{(+)}=\ln \left(\frac{k_{1}-k_{2}}{k_{1}+k_{2}}\right)^{2} .
$$

Let $x_{i c}$ be the center position of the $i$ th soliton. Then, as $t \rightarrow-\infty$, we find

$$
x_{1 c} \sim c_{1} t+x_{10}+\frac{\xi_{10}}{u_{0} k_{1}}, \quad\left(\xi_{1}=\xi_{10}\right) .
$$




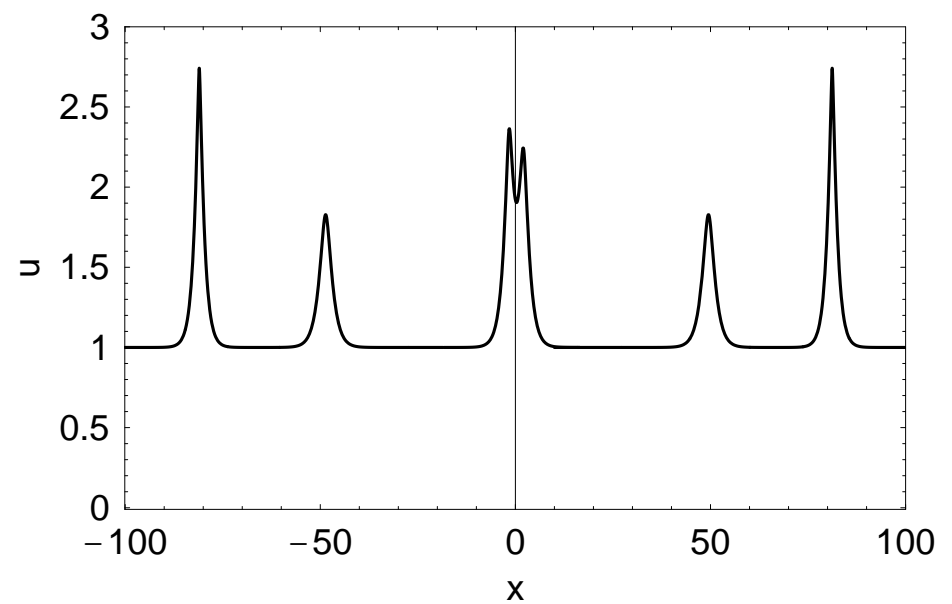

FIG. 3. The interaction of two solitons. $t=-10$ (left: before collision), $t=0$ (middle: during collision), $t=10$ (right: after collision).

As $t \rightarrow+\infty$, on the other hand, $x_{1 c}$ reads

$$
x_{1 c} \sim c_{1} t+x_{10}+\frac{1}{u_{0} k_{1}}\left(\xi_{10}-\delta_{1}^{(+)}\right)-2 \phi_{2}, \quad\left(\xi_{1}=\xi_{10}-\delta_{1}^{(+)}\right) .
$$

The above analysis shows that the asymptotic state of the solution for large time is represented by a superposition of two single solitons in the rest frame of reference. The net effect of the interaction between solitons is the phase shift, which we shall now evaluate. To this end, we define the phase shift of the $i$ th soliton by

$$
\Delta_{i}=x_{i c}(t \rightarrow+\infty)-x_{i c}(t \rightarrow-\infty), \quad(i=1,2) .
$$

Then, we see from (3.20) and (3.21) that the large soliton suffers a phase shift

$$
\Delta_{1}=-\frac{1}{u_{0} k_{1}} \ln \left(\frac{k_{1}-k_{2}}{k_{1}+k_{2}}\right)^{2}-\ln \left(\frac{1+u_{0} k_{2}}{1-u_{0} k_{2}}\right)^{2} .
$$

Performing the similar asymptotic analysis for the small soliton, we obtain the formula for the phase shift. We quote only the final result.

$$
\Delta_{2}=\frac{1}{u_{0} k_{2}} \ln \left(\frac{k_{1}-k_{2}}{k_{1}+k_{2}}\right)^{2}+\ln \left(\frac{1+u_{0} k_{1}}{1-u_{0} k_{1}}\right)^{2} .
$$

The first term of the above formulas is the same as the phase shift arising from the interaction of two solitons for the $\mathrm{KdV}$ equation and the second term comes from the 
mapping (2.7). A new feature appears due to this additional term. An inspection reveals that under the condition $c_{2}<c_{1}\left(k_{2}<k_{1}\right), \Delta_{1}$ is always positive whereas $\Delta_{2}$ takes any sign depending on values of $k_{1}$ and $k_{2}$. Specifically, there exists a critical curve along which $\Delta_{1}=\Delta_{2}$ and beyond which $\Delta_{1}<\Delta_{2}$ so that the small soliton exhibits a larger phase shift than that of the large soliton. It is interesting to observe that the formulas (3.23) and (3.24) coincide formally with those of the two-soliton solution of the $\mathrm{CH}$ equation if one identifies $u_{0}$ with the parameter $\kappa$ which enters into a linear dispersive term $2 \kappa^{2} u_{x}$ in the $\mathrm{CH}$ equation. ${ }^{9}$

The interaction process of two solitons is illustrated in Fig. 3 for three values of time. In this example, $u_{0}=1, c_{1}=0.8, c_{2}=0.5, A_{1}=1.74, A_{2}=0.83$. The phase shifts of the large and small solitons evaluated from (3.23) and (3.24) amount to $\Delta_{1}=2.20$ and $\Delta_{2}=-1.89$, respectively.

\section{C. $N$-soliton solution}

We discuss briefly the asymptotic behavior of the general $N$-soliton solution. First, we order the magnitude of the velocity of each soliton as $0<c_{N}<c_{N-1}<\ldots<c_{1}$ and impose the conditions $0<u_{0} k_{i}<\sqrt{3} / 2,(i=1,2, \ldots, N)$. We take the limit $t \rightarrow-\infty$

with the coordinate $\xi_{i}$ of the $i$ th soliton being fixed. Then, $\xi_{1}, \xi_{2}, \ldots, \xi_{i-1} \rightarrow+\infty$, and $\xi_{i+1}, \xi_{i+2}, \ldots, \xi_{N} \rightarrow-\infty$. The asymptotic forms of $f$ and $f^{\prime}$ from (2.8) and (2.9) are found to be as

$$
\begin{gathered}
f \sim \prod_{1 \leq j<l \leq i-1}\left(\frac{k_{j}-k_{l}}{k_{j}+k_{l}}\right)^{2} \exp \left[\sum_{j=1}^{i-1} \xi_{j}\right]\left(1+\mathrm{e}^{\xi_{i}+\delta_{i}^{(-)}}\right), \\
f^{\prime} \sim \prod_{1 \leq j<l \leq i-1}\left(\frac{k_{j}-k_{l}}{k_{j}+k_{l}}\right)^{2} \exp \left[\sum_{j=1}^{i-1}\left(\xi_{j}-\phi_{j}\right)\right]\left(\mathrm{e}^{\xi_{i}-\phi_{i}+\delta_{i}^{(-)}}\right),
\end{gathered}
$$

where

$$
\delta_{i}^{(-)}=\sum_{j=1}^{i-1} \ln \left(\frac{k_{i}-k_{j}}{k_{i}+k_{j}}\right)^{2} .
$$

Substitution of (3.25a) and (3.25b) into (2.6) and (2.7) yields

$$
u \sim u_{0}+\frac{k_{i}^{2} \tilde{c}_{i}\left\{a_{i} \cosh \left(\xi_{i}-\xi_{i 0}+\delta_{i}^{(-)}\right)+1\right\}}{\left\{\cosh \left(\xi_{i}-\xi_{i 0}+\delta_{i}^{(-)}\right)+a_{i}\right\}^{2}},
$$




$$
x-c_{i} t-x_{i 0} \sim \frac{\xi_{i}}{u_{0} k_{i}}+2 \ln \left(\frac{\sqrt{1-\alpha_{i}} \mathrm{e}^{\xi_{i}-\xi_{i 0}+\delta_{i}^{(-)}}+\sqrt{1+\alpha_{i}}}{\sqrt{1+\alpha_{i}} \mathrm{e}^{\xi_{i}-\xi_{i 0}+\delta_{i}^{(-)}}+\sqrt{1-\alpha_{i}}}\right)-2 \sum_{j=1}^{i-1} \phi_{j} .
$$

In the limit $t \rightarrow+\infty$, on the other hand, $\xi_{1}, \xi_{2}, \ldots, \xi_{i-1} \rightarrow-\infty$, and $\xi_{i+1}, \xi_{i+2}, \ldots, \xi_{N} \rightarrow$ $+\infty$ and the expressions corresponding to (2.25) and (2.26) are given by

$$
\begin{array}{r}
f \sim \prod_{i+1 \leq j<l \leq N}\left(\frac{k_{j}-k_{l}}{k_{j}+k_{l}}\right)^{2} \exp \left[\sum_{j=i+1}^{N} \xi_{j}\right]\left(1+\mathrm{e}^{\xi_{i}+\delta_{i}^{(+)}}\right), \\
f^{\prime} \sim \prod_{i+1 \leq j<l \leq N}\left(\frac{k_{j}-k_{l}}{k_{j}+k_{l}}\right)^{2} \exp \left[\sum_{j=i+1}^{N}\left(\xi_{j}-\phi_{j}\right)\right]\left(1+\mathrm{e}^{\xi_{i}-\phi_{i}+\delta_{i}^{(+)}}\right),
\end{array}
$$

where

$$
\delta_{i}^{(+)}=\sum_{j=i+1}^{N} \ln \left(\frac{k_{i}-k_{j}}{k_{i}+k_{j}}\right)^{2}
$$

and

$$
\begin{gathered}
u \sim u_{0}+\frac{k_{i}^{2} \tilde{c}_{i}\left\{a_{i} \cosh \left(\xi_{i}-\xi_{i 0}+\delta_{i}^{(+)}\right)+1\right\}}{\left\{\cosh \left(\xi_{i}-\xi_{i 0}+\delta_{i}^{(+)}\right)+a_{i}\right\}^{2}}, \\
x-c_{i} t-x_{i 0} \sim \frac{\xi_{i}}{u_{0} k_{i}}+2 \ln \left(\frac{\sqrt{1-\alpha_{i}} \mathrm{e}^{\xi_{i}-\xi_{i 0}+\delta_{i}^{(+)}}+\sqrt{1+\alpha_{i}}}{\sqrt{1+\alpha_{i}} \mathrm{e}^{\xi_{i}-\xi_{i 0}+\delta_{i}^{(+)}}+\sqrt{1-\alpha_{i}}}\right)-2 \sum_{j=i+1}^{N} \phi_{j} .
\end{gathered}
$$

We can see that the asymptotic form of the $N$-soliton solution is a superposition of $N$ single solitons each of which has the form given by (3.4). The phase shift of the $i$ th soliton can be derived from (3.26b) and (3.28b). It reads

$$
\begin{gathered}
\Delta_{i}=\frac{1}{u_{0} k_{i}}\left[\sum_{j=1}^{i-1} \ln \left(\frac{k_{i}-k_{j}}{k_{i}+k_{j}}\right)^{2}-\sum_{j=i+1}^{N} \ln \left(\frac{k_{i}-k_{j}}{k_{i}+k_{j}}\right)^{2}\right] \\
+\sum_{j=1}^{i-1}\left(\frac{1+u_{0} k_{j}}{1-u_{0} k_{j}}\right)^{2}-\sum_{j=i+1}^{N}\left(\frac{1+u_{0} k_{j}}{1-u_{0} k_{j}}\right)^{2}, \quad(i=1,2, \ldots, N) .
\end{gathered}
$$

In the special case of $N=2$, the formulas (3.29) reduce to (3.23) and (3.24). They clearly show that each soliton has pairwise interactions with other solitons, i.e., there are no many-particle collisions among solitons. This feature is common to that of the $N$-soliton solutions of integrable nonlinear PDEs.

\section{CONSERVATION LAWS}


The Bäcklund transformation (2.18) between solutions $p$ and $q$ of the two integrable equations allows us to construct an infinite number of conservation laws of the $\mathrm{mCH}$ equation in a simple manner. To demonstrate this, we first note that the SWW equation (2.15) exhibits local conservation laws of the form

$$
w_{n, \tau}=j_{n, y}, \quad(n=0,1,2, . .),
$$

where the conserved density $w_{n}$ and associated flux $j_{n}$ are polynomials of $q$ and its $y$ derivatives. We rewrite (4.1) in terms of the variables $x$ and $t$ by using (2.1). Substituting Eq. (1.1) into the resultant equation, we obtain

$$
\left(m w_{n}\right)_{t}=\left[j_{n}-m\left(u^{2}-u_{x}^{2}\right) w_{n}\right]_{x}
$$

It turns out that the quantities

$$
I_{n}=\int_{-\infty}^{\infty} m w_{n} d x, \quad(n=0,1,2, \ldots),
$$

become the conservation laws of the $\mathrm{mCH}$ equation if one introduces $q$ from (2.18) into $w_{n}$.

The conservation laws of the SWW equation take the same form as those of the KdV equation since both equations belong to a common integrable hierarchy. For completeness, we reproduce them shortly. We start from the Lax pair associated with the SWW equation $^{20}$

$$
\begin{gathered}
\psi_{y y}-q \psi=\frac{\lambda}{u_{0}^{2}} \psi, \\
(4 \lambda-1) \psi_{\tau}=2 u_{0}^{2}\left(u_{0}+\partial_{y}^{-1} q_{\tau}\right) \psi_{y}-u_{0}^{2} q_{\tau} \psi,
\end{gathered}
$$

where $\lambda$ is a spectral parameter. If we put $w=\psi_{y} / \psi$, we can rewrite (4.4) into the form

$$
\begin{gathered}
w_{y}+w^{2}-q=\frac{\lambda}{u_{0}^{2}}, \\
(4 \lambda-1) w_{\tau}=\left[2 u_{0}^{2}\left(u_{0}+\partial_{y}^{-1} q_{\tau}\right) w-u_{0}^{2} q_{\tau}\right]_{y} .
\end{gathered}
$$

Thus, the quantity $\int_{-\infty}^{\infty} w d y$ is conserved in $\tau$. Expanding $w$ in powers of $\epsilon\left(\equiv u_{0} /(2 \sqrt{\lambda})\right)$ as $w=1 /(2 \epsilon)+\sum_{n=1}^{\infty} \epsilon^{n} w_{n}$ and substituting it into (4.5a), we obtain, after equating the 
coefficients of $\epsilon^{n}$, the recursion relation that determines $w_{n}$

$$
w_{n+1}=w_{n, y}-\sum_{m=1}^{n-1} w_{n-m} w_{m}, \quad(n \geq 2),
$$

with the initial conditions $w_{1}=q$ and $w_{2}=-q_{y}$. We quote a few conserved densities:

$$
w_{3}=q_{y y}-q^{2}, \quad w_{4}=\left(-q_{y y}+2 q^{2}\right)_{y}, \quad w_{5}=\left(q_{y y y}-6 q q_{y}\right)_{y}+2 q^{3}+q_{y}^{2}
$$

The quantity $q$ from (2.18) can be rewritten in terms of $m$ as

$$
q=-\frac{m_{y}}{2 m^{2}}+\frac{1}{4 m^{2}}-\frac{1}{4 u_{0}^{2}}
$$

Last, substituting (4.7) with (4.8) into (4.3) and taking into account the relation $\partial / \partial y=m^{-1} \partial / \partial x$, we obtain a sequence of conservation laws of the $\mathrm{mCH}$ equation expressed in terms of the original spatial variable. We see that $w_{n}$ for even $n$ takes the form of a perfect derivative $\left(W_{n}\right)_{y}$ and hence the quantity $I_{n}$ for even $n$ vanishes identically. Actually, $\int_{-\infty}^{\infty} m\left(W_{n}\right)_{y} d x=\int_{-\infty}^{\infty}\left(W_{n}\right)_{x} d x=0$. Hence, only the quantities $I_{n}$ for odd $n$ survive. We can express the nontrivial conservation laws as $I_{2 n+1}=\sum_{m=0}^{n+1} \nu_{n m} \tilde{I}_{m}$, where $\nu_{n m}$ are constants depending on $u_{0}$. The first four of $\tilde{I}_{n}$ read as follows:

$$
\begin{gathered}
\tilde{I}_{0}=\int_{-\infty}^{\infty}\left(m-u_{0}\right) d x \\
\tilde{I}_{1}=\int_{-\infty}^{\infty}\left(\frac{1}{m}-\frac{1}{u_{0}}\right) d x \\
\tilde{I}_{2}=\int_{-\infty}^{\infty}\left[\frac{1}{m^{3}}-\frac{1}{u_{0}^{3}}+4 \frac{m_{x}^{2}}{m^{5}}\right] d x \\
\tilde{I}_{3}=\int_{-\infty}^{\infty}\left[\frac{1}{m^{5}}-\frac{1}{u_{0}^{5}}+8 \frac{m_{x x}^{2}}{m^{7}}+20 \frac{m_{x}^{2}}{m^{7}}-56 \frac{m_{x}^{4}}{m^{9}}\right] d x .
\end{gathered}
$$

Note that $\tilde{I}_{0}$ follows directly from the $\mathrm{mCH}$ equation (1.1).

Last, we remark that an infinite number of conservation laws have also been obtained quite recently using the fact that the $\mathrm{mCH}$ equation describes pseudo-spherical surfaces ${ }^{21}$ which is equivalent to the existence of the Lax representation for the $\mathrm{mCH}$ equation. ${ }^{5} \mathrm{On}$ the other hand, our derivation is based on a purely algebraic procedure relying on the Bäcklund transformation. 


\section{CONCLUDING REMARKS}

In this paper, we have developed a systematic procedure for constructing smooth soliton solutions of the $\mathrm{mCH}$ equation. In the process, the associated $\mathrm{mCH}$ equation has played a central role which has been derived from the $\mathrm{mCH}$ equation via a reciprocal transformation. It is important that certain condition must be imposed on the soliton parameters to assure the smoothness of the solutions. Specifically, a detailed inspection of the one-soliton solution reveals that there exists a critical value of the amplitude parameter beyond which the solution becomes a many-valued function. This feature differs from that of the one-soliton solution of the $\mathrm{CH}$ equation for which the peakon solution is produced from the smooth soliton solution in the limit of small dispersion. ${ }^{18,19}$ Whether the singular solutions such as the W/M-shape soliton and cusp soliton found by Qiao ${ }^{5}$ can be obtained from smooth solitons by means of appropriate limiting procedure is an interesting issue to be studied in a separate context.

An exact method of solution presented here is also applicable to a variant of the $\mathrm{mCH}$ equation, for example,

$$
m_{t}+\left[m\left(u^{2}-u_{x}^{2}\right)\right]_{x}+\gamma u_{x}=0, \quad m=u-u_{x x}
$$

where $\gamma$ is a real parameter. When $\gamma=0$, this equation is shown to exhibit peakons under the boundary condition $u \rightarrow 0$ as $|x| \rightarrow \infty .^{22}$ In addition, another version of the $\mathrm{mCH}$ equation with cubic nonlinearity

$$
m_{t}+u^{2} m_{x}+3 u u_{x} m=0, \quad m=u-u_{x x}
$$

attracts an interest because of its different mathematical structure from that of the $\mathrm{mCH}$ equation under consideration. This equation has been discovered by Novikov ${ }^{23}$ and admits a Lax pair related to a negative flow of the Sawada-Kotera hierarchy through a reciprocal transformation. ${ }^{24}$ For both equations (5.1) and (5 .2), we obtained smooth single soliton solutions which satisfy the boundary condition $u \rightarrow u_{0}$ as $|x| \rightarrow \infty$. However, the general $N$-soliton problem still remains open and it reserves a future study.

In conclusion, we comment on a paper by Ivanov and Lyons. ${ }^{8}$ Applying the IST to an initial value problem of the $\mathrm{mCH}$ equation under the boundary condition $m \rightarrow m_{0}\left(=u_{0}\right)$ 
as $|x| \rightarrow \infty$, they presented the explicit parametric representations of the one- and twosoliton solutions for the quantity $m\left(=u-u_{x x}\right)$ which take the form of dark solitons. See Fig. 1 in their paper, for example. However, the expression (37) for $x$ is incorrect. Actually, the sign of the first term on the right-hand side of (37) should be minus in place of plus. This discrepancy has been caused by an error in Eq. (32). Specifically, a factor

$\mathrm{e}^{i k y}$ should read $\mathrm{e}^{-i k y}$. The same error happened in Eq. (37) as well for the two-soliton solution. If these errors were corrected appropriately, then their result would coincide with that given in the present paper. Note, however that Ivanov and Lyons obtained the expression of $m$ only. On the other hand, we have a compact parametric representation of $u$ for the first time.

\section{ACKNOWLEDGEMENTS}

This work was partially supported by JSPS KAKENHI Grant Number 22540228. 


\section{REFERENCES}

1. A.S. Fokas, The Korteweg-de Vries equation and beyond, Acta. Appl. Math. 39, 295-305 (1995).

2. B. Fuchssteiner, Some tricks from the symmetry-toolbox for nonlinear equations: Generalizations of the Camassa-Holm equation, Phys. D95, 229-243 (1996).

3. P.J. Olver and P. Rosenau, Tri-Hamiltonian duality between solitons and solitarywave solutions having compact support, Phy. Rev. E53, 1900-1906 (1996).

4. R. Camassa and D.D. Holm, An integrable shallow water equation with peaked solitons, Phy. Rev. Lett. 71, 1661-1664 (1993).

5. Z. Qiao, A new integrable equation with cuspons and W/M-shape-peaks solitons, J. Math. Phys. 47, 112701 (2006).

6. D.D. Holm and R. Ivanov, Smooth and peaked solitons of the CH equation, J. Phys. A: Math. Theor. 43, 434003 (2010).

7. Z. Qiao, New integrable hierarchy, its parametric solutions, cuspons, one-peak solitons, and M/W-shape peak solitons, J. Math. Phys. 48, 082701 (2007).

8. R.I. Ivanov and T. Lyons, Dark solitons of the Qiao's hierarchy, J. Math. Phys. 53, 123701 (2012).

9. Y. Matsuno, Parametric representation for the multisoliton solution of the CamassaHolm equation, J. Phys. Soc. Jpn. 74, 1983-1987 (2005).

10. Y. Matsuno, Multisoliton solutions of the Degasperis-Procesi equation and their peakon limit, Inverse Prob. 21, 1553-1570 (2005).

11. Y. Matsuno, The $N$-soliton solution of the Degasperis-Procesi equation, Inverse Prob. 21, 2085-2101 (2005).

12. M.J. Ablowitz, D.J. Kaup, A.C. Newell and H. Segur, The inverse scattering transform - Fourier analysis for nonlinear problems, Stud. Appl. Math. 53, 249-315 (1974). 
13. T.L. Perelman, A.Kh. Fridman and M.M. El'yashevich, On the relationship between the $N$-soliton solution of the modified Korteweg-de Vries equation and the KdV equation solution, Phys. Lett. 47A, 321-323 (1974).

14. H. Ono, Solitons on a background and a shock wave, J. Phys. Soc. Jpn. 40, 1487-1496 (1976).

15. R. Hirota and J. Satsuma, $N$-soliton solutions of model equations for shallow water waves, J. Phys. Soc. Jpn. 40, 611-612 (1976)

16. R.M. Miura, Korteweg-de Vries equation and generalizations I. A remarkable explicit nonlinear transformation, J. Math. Phys. 9, 1202-1204 (1968).

17. R. Hirota, Direct methods in soliton theory. In Solitons (ed. R.K. Bullough and P.J. Caudrey, Springer-Verlag, New York, 1980) 157-176.

18. A. Parker and Y. Matsuno, The peakon limits of soliton solutions of the CamassaHolm equation, J. Phys. Soc. Jpn. 75, 124001 (2006).

19. Y. Matsuno, The peakon limit of the $N$-soliton solution of the Camassa-Holm equation, J. Phys. Soc. Jpn. 76, 034003 (2007).

20. P.A. Clarkson and E.L. Mansfields, On a shallow water wave equation, Nonlinearity 7, 975-1000 (1994).

21. P.M. Bies, P. Górka and E.G. Reyes, The dual modified Korteweg-de Vries-FokasQiao equation: Geometry and local analysis, J. Math. Phys. 53, 073710 (2012).

22. G. Gui, Y. Liu, P.J. Olver and C. Qu, Wave-breaking and peakons for a modified Camassa-Holm equation, Commun. Math. Phys. (2012), 319, 731-759 (2013).

23. V. Novikov, Generalizations of the Camassa-Holm equation, J. Phys. A: Math. Theor. 42, 342002 (2009).

24. A.N.W. Hone and J.P. Wang, Integrable peakon equations with cubic nonlinearity, J. Phys. A: Math. Theor. 41, 372002 (2008). 